\title{
Exploring professional issues: the psychosocial component of genetic counseling in genomic healthcare
}

\author{
Caroline Benjamin*,1(D), Alan Phillips², Julia Finch¹, Louise Dubois', Lisa McGrath¹, Claire \\ Kulke ${ }^{1}$, Claire Brooks ${ }^{1}$, Pam Harris ${ }^{1}$, Janette Daly ${ }^{1}$, Jan Birch ${ }^{1}$ \& Katie Nickson ${ }^{3}$ \\ ${ }^{1}$ Liverpool Centre for Genomic Medicine, Liverpool Women's NHS Foundation Trust, Liverpool L8 7SS, UK \\ ${ }^{2}$ Alan Phillips Associates, Lancashire, England, UK \\ ${ }^{3}$ Academic Unit of Medical Genetics \& Pathology, University of Glasgow, Glasgow, Scotland, UK \\ *Author for correspondence: Benjaminc@cardiff.ac.uk
}

\begin{abstract}
Aim: To determine UK genetic counselors' (UKGCs) opinion regarding 'the psychosocial component of the UKGC remit in the new genomics era'. Methods: Facilitated discussions at a national conference (2016) using interactive methodologies (58 participants). Results: UKGCs recognized the rapid rate of change emerging with advances in genomic science. Change will be required to the UKGC remit and the roles, rules, relationships and responsibilities that underpin it (29 topics identified). UKGCs supported their 'unique selling point'; integrating knowledge and the explicit focus on psychosocial aspects of genomic healthcare. By 2019, some of the aspirations have been achieved. Conclusion: UKGCs should proactively position themselves to capitalize on the challenges and opportunities of genomic healthcare to maximize patient benefit.
\end{abstract}

First draft submitted: 15 April 2019; Accepted for publication: 17 September 2019; Published online: 10 October 2019

Keywords: genetic counseling • genomics $\bullet$ workforce

The Association of Genetic Nurses and Counselors (AGNC) is the largest professional group representing genetic counselors in the UK and the Republic of Ireland [1]. Their 2017 statement, 'the future role of genetic counselors in genomic healthcare' identifies the importance of continuing professional education and responding to new ways of working. Genetic counselors globally are facing rapid technological challenges and evidence suggests that they are changing practice to meet healthcare needs [2]. UK healthcare policy has embraced the benefits of genomic technologies and personalized medicine with the funding of large scale change projects such as the 100,000 Genomes Project [3] and the central role of genomics within the UK Chief Medical Officers 2016 Report 'Generation Genome', culminating in 2017 with the intent to establish an NHS Genomics Medicine Service [4]. However, recent research has indicated there are difficulties to overcome regarding implementation of such a service, combining both research objectives and clinical care [5]. There are also challenges faced by attempting to introduce genetic testing into mainstream (i.e., non clinical genetic) clinical speciality care, known as 'mainstreaming' within UK healthcare policy [6].

This paper describes empirical research undertaken in 2016 and provides an insight into UK genetic counselors' (UKGCs) opinions about their current role and future opportunities and challenges. The aim of this research was to determine UKGCs opinion regarding 'the psychosocial component of the UKGC remit in the new genomics era' at a national meeting. Also to revisit and discuss, in this paper, the evidence regarding current progress on issues which were raised and highlight the important role UKGCs have in driving forward the integration of genomic medicine. This is of interest to the international community of genetic counselors to provide a UK perspective. In addition, due to the expansion of multidisciplinary working, it is of importance to the extended healthcare team; clinical geneticists, clinical scientists, psychologists, clinical specialists policy makers and managers of clinical services. 
The UK NHS is a universal healthcare system which provides free or low-cost healthcare to all legal residents of the UK and is funded through taxation. Nearly all UKGCs work within the NHS Clinical Genetics Service, a network of centers commissioned to provide a set of services as defined by service specification documents [7]. The UK NHS is under unprecedented financial constraint, continually striving to improve quality and reduce costs. The NHS five year forward view called for efficiency saving of $£ 22$ billion and many service managers are under pressure to improve waiting times [8].

UKGCs, at $0.05 \%(330 / 750,000)$ make up an incredibly small proportion of the UK NHS clinical workforce, this is in comparison with nurses and midwives who comprise $42.67 \%(320,000 / 750,000)$ and healthcare scientists $6.66 \%(50,000 / 750,000)$ [9-11]. They do however have a unique skill set which could be pivotal in moving forward with implementing genomic technologies to improve patient care. UKGCs provide patient facing contact, as well as education and support for the wider healthcare community who use genetic/genomic information in their practice. Resta et al. defined the process of genetic counseling as, 'advising individuals and families affected by, or at risk of, genetic disorders to help them understand and adapt to the medical, psychological and familial implications of genetic contributions to disease. The process integrates: Interpretation of family and medical histories to assess the chance of disease occurrence or recurrence: Education about inheritance, testing, management, prevention, resources and counseling to promote informed choices and adaptation to the risk or condition' [12]. More recently, the AGNC have developed a framework to help describe the genetic counselor's role in the implementation of genomics, which includes a broader range of roles than traditionally encompassed by Resta $e t$ al. [2].

The debate continues regarding the level of counseling skills required by genetic counselors and the centrality of the counseling activity to the effectiveness of genetic counseling sessions. Austin et al. reviewed available evidence, although lacking, from process and outcome studies, which showed that, 'genetic counseling is a therapeutic encounter that cannot be reduced to one where the counselor performs a simple 'conduit for information' function, without losing effectiveness' [13]. The literature advocating similarities between genetic counseling and therapeutic counseling is contentious and has been the focus of a recent debate within the US genetic counseling profession with Biesbecker, Austin and Caleshu [14] proposing that 'psychotherapeutic genetic counseling' is a genetic counseling practice discipline and Redlinger-Grosse, arguing that, "psychosocial counseling for genetic counselors has a different process (e.g., brief, present focused), as well as a more specific outcome goal (e.g., decision-making, acceptance of genetic diagnosis and communication of genetic information) than interactions in psychotherapy" [15]. The literature predominantly reflects perspectives from the USA, whose healthcare systems and national cultures are significantly different to those of the UK NHS, and therefore, translating these findings into a UK setting does not take sufficient account of important contextual factors specific to genetic counseling in a public sector health service.

The counseling skills training genetic counselors receive in the UK, and the time they are allocated for patient care, does not intend to equip them to practice to the same registration standards as UK-accredited psychotherapists (British Association of Counselors and Psychotherapists (BACP) [16] and UK Council for Psychotherapy (UKCP) [17]). The professional role of the UKGC is impacted by contextual considerations (i.e., political, economic, social, technological, legal and environmental) and these contextual factors have important ethical implications for the 4Rs (i.e., the role, rules, relationships and responsibilities) of ethical genetic counseling practice [18,19]. Author AP developed the 4Rs ethical practice framework for the Skilled Helper Programme (SHP) for healhtcare professionals to assist in boundary role management, reflective practice and continuing professional development (CPD) of multidisciplinary professionals. The 4Rs has since been adapted for UKGCs and is the basis of the bespoke model of genetic counseling consultative supervision and CPD that has been adopted by several GC teams in the UK [20].

The AGNC Job Profiles currently recommend 45-60 min for a consultation [4] and often practitioners, outside of predictive testing sessions, are restricted to one or two sessions per client. In some contexts, UKGCs have acknowledged that "all too often, because of time and other resource pressures, we are left with feeling that the consultation was less than satisfactory, that we have let clients down" [21]. The contextual factors that make genetic counseling a form of 'time-sensitive' counseling are essential considerations, as it is acknowledged that "time-limited practice done badly is probably worse than longer time work done badly (as) the time-limited practitioner has only the moment to facilitate change, and may not have the time to recover from technical mistakes that is available to longer time workers" [22]. While most healthcare professionals use 'counseling' in the form of generic, 'embedded' skills; in the UK NHS, UKGCs are the only NHS healthcare professionals whose training, qualifications and registration criteria includes psychosocial counseling. As such, in business and promotional terms, this psychosocial component of genetic counseling represents a 'unique selling point' (USP). 
Educational preparation in the UK for genetic counselors includes a number of routes; including Masters level genetic counselor and Degree level nursing training programmes, which equip practitioners who are then further assessed as having attained competencies in genetics and counseling skills. In the UK, the profession of genetic counseling has evolved and expanded over a number of years and most genetic counselors are now registered with the UK and Republic of Ireland Genetic Counselor Registration Board (GCRB) [23].

A recent global overview of the genetic counseling profesion reported variation between countries. Across Europe, in many countries, it is physicians who primarily provide genetic counseling. However, as genomic healthcare advances, genetic counselors and nurses in other European countries continue to have a strong influence in the provision of genetic counseling to patients [24]. It is estimated that there are currently nearly 7000 genetic counselors across 28 countries [24-26], the majority being located in North America. Recent commentatory suggests that there is an expanding role for genetic counselors within the US precision healthcare inititives [26]. The rapid development of scientific knowledge and technologies has enhanced the ability of genomic data to inform healthcare and with it, increased the complexity of test implementation and interpretation for healthcare professionals and patients [27].

\section{Methods}

At the 2016 AGNC annual conference, participants considered the future of genetic counseling in a workshop (2 h), attended by all conference participants, entitled 'the psychosocial component of the genetic counseling remit in the new genomics era'. The workshop comprised of an initial plenary presentation session delivered by author AP followed by splitting the delegates into focus groups and then reconvening for a final group discussion [28]. The focus group approach was chosen because of its appropriateness in time-limited contexts. The sharing of views, experiences and stories during a focus group is considered advantageous in providing insightful and rich data which may be overlooked in the more private and solitary forms of data gathering, such as by questionnaire [20].

The AGNC conference was advertised primarily through the AGNC website. Participants at the conference (60) were self-selected and the majority of conference participants attended the workshop (58). Therefore this was a convenience sample, as participants were selected by their attendance at the workshop. The majority were GCRB Registered Genetic Counselors (32), nonregistered genetic counselors/genetic nurses (21), genetic counselors from other countries (3), researchers (3) and a trainee medical clinical geneticist (1). The participants represented a wide range of clinical genetics centers across the UK (20 out of the 23 centers were represented), including England, Scotland and Wales. The delegates were split into five groups (maximum 12 people) and each facilitated session lasted $1 \mathrm{~h}$. All the groups were facilitated by genetic counselors from the organizing committee of the meeting and all had experience in facilitating group work. Discussion centerd around four preprepared questions which had been developed between author AP and the facilitation team in advance of the meeting.

\section{Question 1}

How relevant will the counseling component of genetic counseling be in an era of genomic medicine and associated health economy dominated by the scientific, managerial and mainstreaming agendas?

\section{Question 2}

Is the counseling component of the genetic counseling remit something to be valued and maintained?

\section{Question 3}

If the counseling component of genetic counseling is valuable enough to be protected as a unique activity and selling point in this health economy, what strengths, weaknesses, opportunities and threats (SWOT) do these forces of change pose?

\section{Question 4}

What will genetic counselors have to keep, introduce, develop and stop (KIDS) to equip them to meet the needs of commissioners, employers and service users in a market led health economy among the market forces of competing resources and health activities?

In the facilitated sessions, each question was presented in turn on a flip chart and the facilitator noted on the flip chart the main topics which were raised. The facilitator managed the time, maintained focus and guided participants to document their responses. In the final group discussion, participants were asked to place their comments (on 
Table 1. Strengths, weaknesses, opportunities and threats analysis: 18 topics identified.

Strengths

- Unique selling point

- The protected status of genetic counseling

- Knowledge and experience

- Training and expertise

- Psychosocial counseling

$-\mathrm{CPD}$

- Genomics, counseling and mainstreaming

- A diverse profession

- Gatekeeping

Threats and weaknesses

- Gatekeeping and mainstreaming

- Perceptions, understanding and misunderstanding

- Performance measurement and value and management pressure

- Mainstreaming, bioinformatics, consent and ethical practice

- Private direct to consumer testing

CPD: Continuing professional development.
Opportunities

- The size of profession

- Promotion and championing of genetic counseling

- Research and recruitment

- Mainstreaming and educating

\section{Table 2. Keep, introduce, develop and stop analysis: 11 topics identified.}

\section{Keep and develop}

- Professional identity and distinctiveness

- Education and supervision

- Teaching and education

- Patient focus and time

- Multidisciplinary working

Introduce

- Positioning and continuing professional development

- More multidisciplinary working

- Educating, publicity and marketing

Stop

- Resistance to change

- Practitioner self-care/responsibility:

- Overcoming Isolation and multidisciplinary working

sticky notes) onto two poster boards which were laid out in a grid using the SWOT and KIDS interactive group techniques:

SWOT (Strengths, Weaknesses, Opportunities and Threats) and KIDS (Keep, Introduce, Develop and Stop). The SWOT tool provides a manageable mechanism for balancing the pros and cons associated with the topic under discussion [29]. The KIDS tool is described as an 'effective and dynamic way of conducting an exercise, as a simple way of quickly diagnosing or assessing the view of a team about the activities, projects or issues within a department or organization'[30]. It offers a manageable means of generating discussion, it collates future-oriented views regarding what and how the group thought they could influence the future direction of their profession [31,32]. The focus groups were preceded by an initial plenary session which used the PESTEL framework to consider the 'Political, Economic, Social, Technological, Environmental and Legal' drivers and influences on the current and future remit of genetic counseling, this framework enhances the SWOT and KIDS techniques [33]. These interactive tools have been proved successful in the clinical supervision and CPD of UKGCs [18]. When dealing with a potentially broad and complex subject such as this, in the limited time available, the SWOT and KIDS tools were chosen as they are recognized as efficient and effective tools for generating discussion, focusing attention and managing the time and process.

Delegates were asked if anonymized data from the focus group could be collected and used in a research project, all verbally agreed prior to the start of the workshop. When conducting this research, the principles outlined in the Declaration of Helsinki were followed and institutional review was not required.

\section{Results \& analysis}

The data obtained from the sticky notes and flip charts, as well as written notes of the discussion at the final plenary session were collated and discussed by the facilitators a few months after the conference. Eighteen topics were identified by SWOT analysis and 11 topics by KIDS analysis. Text marked with quotation marks below indicate a direct quote from a workshop participant and no priority has been afforded to the order in which the themes are presented (Table $1 \& 2$ ).

The 29 topics were then discussed by a subgroup of the authorship team and synthesized into five themes. The analytic framework used for this stage was that of identifying key concepts [34], where the evidence relating to each 
topic was reviewed and similarities and differences were explored, with the aim of identifying key concepts. This iterative technique led to the development of five themes.

Theme 1 - Identity: professional role \& the value of genetic counselors

Participants demonstrated that there was overwhelming agreement that genetic counselors should maintain their existing identity as a distinct professional group. There was strong agreement that genetic counselors should keep a sense of pride and unity. The participants valued the protected status of their profession in the UK and their status as autonomous practitioners. Participants felt genetic counselors and patients benefited from the recruitment of genetic counselors from a diverse background including nursing, scientific and the MSc in genetic counseling routes. Participants acknowledged that in the UK, genetic counseling is a small profession in terms of numbers and service centers. This could be seen as both a weaknesses/threat or as a strength/opportunity, depending on how this was approached.

Participants recognized the difficulties involved in demonstrating the value of genetic counseling and measuring what they do as a way of proving the value of the counseling element of healthcare. There was a strong sense that many of the professionals that genetic counselors liaise with, are from a scientific background, and these individuals may place less importance on the counseling element of the genetic counselor role. They perceived that generally there was a misunderstanding of the process of genetic counseling and what genetic counselors do. This was seen as a weakness/threat and that work to improve this was an important development need for the profession.

There was a strong sense that the AGNC and GCRB should be championing the importance of the role of genetic counselors and the importance of the psychosocial component of their remit. Participants also acknowledged that they should take the opportunity to promote the profession by becoming more involved in research and recruitment and through publishing work. The need to develop a pro-active national publicity and marketing strategy was identified. It was felt that much greater awareness of genetic counseling could be achieved by aligning UKGCs with and utilizing the work done by, Health Education England and the UK 100,000 Genome Project and other public education agencies.

"We should be developing stronger national working groups such as prenatal and cardiac groups that are Genetic Counselor led".

"We have the ability to offer patients time and space to think about their decisions and the implications this may have for their families".

"... with new technologies emerging, GCs are already well educated in this area and therefore, already have the knowledge and skills of genomics".

\section{Theme 2 - Families: a flexible approach}

Participants valued the family and the psychosocial aspects involved in the genetic counseling remit.

A high value was placed on counseling skills and the benefits to patients and families from the counselors' commitment to CPD of their skills. The gatekeeping role of the job was seen as valuable: as gatekeepers of genetic testing, we ensure that people are prepared for a test result and the implications for themselves and wider family members. Concern was expressed regarding the increased pressure from managers regarding length of appointments and the number of patients seen per clinic. In some centers, genetic counselors felt an increasing pressure from their hospitals to see more patients, over shorter appointments and to complete the patient consultation in one session. Some participants felt this would leave no time to explore the psychosocial issues that arise for many patients.

In particular, a major topic was the concern that the counseling component of a session may be deemed a luxury by non genetic counselor colleagues. In terms of effectiveness and quality: it was acknowledged that this would only be possible if appropriate time was maintained to keep our appointment length and pretest counseling. Promotion of patient-centerd care was aligned with the theme of developing and increasing genetic counselor presence on national working groups, for example, the UK Foetal Genomics Group and the UK Association of Inherited Cardiac Conditions.

"Our focus is on the family unit as well as the individual when necessary".

"A flexible approach that enables us to offer additional appointments if necessary".

"Experience of working with family conflicts". 
Theme 3 - The USP: counseling skills

Participants felt that Genetic counseling is a distinct profession which has a USP - that of an explicit focus on psychosocial aspects of genomic healthcare; it is this that distinguishes it from other professions. Participants highlighted that their combination of training in both science and psychosocial counseling as a particular strength. This is in contrast to other genetics professionals where an emphasis is often placed on the scientific and medical aspects of consultations. Their protected status, autonomy and USP are associated with the distinct knowledge, experience and training that equips them as genetic counselors. The importance of the role as educators and communicators was also stressed; they considered themselves to be a valued profession and this was due in large part to the time they are able to offer patients.

Genetic counselors felt the need to promote this USP as a strategy for educating government, managers, commissioners and service providers. They wished to highlight the valuable and distinct role of the genetic counseling profession.

"We use our counseling and communication skills in interpreting complex information".

"Genetic counselors have a role in preventing post-decision-regret".

"We should incorporate psychotherapeutic counseling into the GC repertoire".

\section{Theme 4 - Movement into the mainstream}

The importance of the genetic counselor as a bridge between genomic science and its interpretation to patients and other professionals was a major theme. This was deemed a significant strength with regard to the UK policy mainstreaming (moving genetic healthcare into diverse clinical specialties and primary care) agenda. The training that genetic counselors receive in counseling skills, along with their knowledge, ideally equips them to be the lead trainers in the mainstreaming and associated agendas, if they promote and market themselves effectively. There was common agreement that genetic counselors should continue developing their teaching and education roles as part of the mainstreaming agenda but with greater emphasis on genetic counselor led education programmes. This was considered important for patients and as a means of raising genetic counselor visibility and status.

Although the mainstreaming role was seen as one of the strengths of UKGCs, some delegates thought that mainstreaming would relinquish the control that they currently felt they had and this would be detrimental to patients and families. Notwithstanding the strengths and opportunities associated with the mainstreaming agenda, significant concerns were expressed about the lack of training other health professionals received in taking consent for genetic tests. In particular, the worry was that the counseling component would be lost when there is lots of other scientific information already being provided; that, mainstreaming would potentially make patients less prepared for results and that there would be an increase in the number of patients seen post-testing. Participants recognized that while they were ideally equipped to provide mainstreaming training and minimize the aforementioned weaknesses and threats associated with mainstreaming, they were equally concerned that they might not be allocated the appropriate amount of time needed to train other professionals adequately. Therefore, other health professionals would not have appropriate counseling skills training and may not incorporate the counseling component into their appointments. Concerns were voiced in respect of the implications of this for patient consent.

".. mainstreaming would potentially make patients less prepared for results".

"I would be concerned that there would be an increase in the number of patients genetic counselors would see for [only] post-test counseling".

"Genetic Counselors need to exchange our skills with others".

\section{Theme 5 - Change: future working practices}

Keeping multidisciplinary working was seen as important and this was something that needed further development for patients and professionals alike. It was felt that more could be done to share and exchange skills through regular case discussion with other GCs and other professionals. Such as sharing standardized information; leaflets, letters, pathways and proformas. Participants wanted to work in multidisciplinary clinics such as neurology, cardiology and specialist cancer. There was also a need to investigate closer working relationships with UK charities such as the Huntington Disease Association and Unique. There was a strong sense that UKGCs should stop working in isolation as genetic counselors and genetic counseling teams, as stronger alliances with other professionals may help some of the pressures. It was recognized that genetic counseling would have to develop new and innovative ways of counseling and communicating with patients and professionals by embracing technology such as Skype, 
teleconferencing and communication applications (apps). Participants acknowledged that they would have to resist feeling 'closed' minded about the future; worrying too much and feeling too threatened by the forces of change.

The potential rise in direct to consumer private genetic testing services was considered a significant threat. Private direct to consumer providers do not have to follow the same 'rules' (NHS policies, protocols) as most UKGCs. Concern was expressed equally for; the threat to the profession, remit of genetic counseling and for patients. This filtered into the above discussion of consent and ethics, as it was seen as a potential detrimental threat to patients who would not receive the same amount and quality of pretest counseling.

"GCs need to equip themselves for different counseling (styles) and embrace communication technology".

"GCs need to exchange our skills and need regular case discussion".

This research has limitations in that it was a convenience sample of UKGCs and may not have been representative of the UK scope of genetic counselor practice. The methodology and analysis strategy used was only developed to highlight key concept areas, rather than be a more in depth thematic analysis. The data were collected in 2016 and since that time the English 100,000 Genomes Project has ended and a new NHS Genomic Medicine Service developed and it would be helpful to revisit these questions in a few years.

\section{Discussion}

The results from this research show that UKGCs recognize the scope and range of change that will continue to emerge with advances in genomic medicine and policy agendas. They acknowledged that advances in technology will require changes to the genetic counseling remit and the role, rules, relationships and responsibilities that underpin it. There was debate regarding how to respond to the changes and how to position the community to exercise as much influence as possible over them. Enthusiasm was generated regarding the genetic counselor 'unique selling point' (USP); in particular, their knowledge, training and the explicit focus on the psychosocial aspects of genomic healthcare. There was debate regarding how to respond to the changes and how to position the UKGC community to exercise as much influence as possible over them.

This research is significant as it presents empirical evidence from a group of UKGCs into the perceived strengths and weaknesses of the UKGC profession. Specifically UKGCs hopes and concerns for the future at the start of the 'genomic medicine era'. It provides a basis with which to compare the experiences and perceptions of UKGCs with genetic counselor colleagues in other countries and other healthcare systems, as the profession of genetic counseling continues to expand globally [24]. We discuss how the UKGC profession has developed in 3 years since this research and what change has already been implemented.

Since the workshop in 2016, some of the aspirations mentioned by participants have been achieved and some challenges remain. September 2016 marked the launch of a new training route in England into the genetic counseling profession, the 'Scientist Training Programme (STP): Genomic Counseling'. Genetic counselors were pivotal in working together with Health Education England (Genomics England) and the National School of Healthcare Science to secure funding for UKGC training places and in developing the curriculum of this course. Members of the UKGC profession continue to develop the Scientific Training Programme and act as Training Officers and Assessors for the Scientist Training Programme Genomic Counseling students [35]. Over the last few years, papers in prominent scientific journals feature work by UKGCs. In 2017, the AGNC published a position statement outlining the role of genetic counselors in genomic healthcare in the UK which concluded 'genetic counselors are a highly qualified and skilled workforce in a very strong position to make great contributions to the mainstreaming of genomics' [2]. A recent invited review of the global status of genetic counselors, highlights many of the themes presented by our research findings and describes the current focus of genetic counseling in the genomic era [25]. Following the 2016 meeting of the Transnational Alliance of Genetic Counseling (an organization established to foster communication within the international genetic counseling community), UKGCs worked with colleagues to publish their findings on the state of the genetic counseling profession, with a focus on educational preparation and workforce [26]. This paper describes a profession well established in some countries through to nonexistent in others.

Of concern to some participants of this research in 2016 was the challenge of maintaining the quality of the psychosocial element of the counseling relationship, while providing a service within the increasingly constrained financial environment of the UK NHS. The AGNC have recently reviewed the genetic counselor job plans, recommending that genetic counselor sessions remain between 45 and 60 min in length and that no more than four families are seen during a standard half-day clinic session [36]. Joseph et al. (2018) voices her concern in an opinion piece that in a resource limited UK NHS, the psychosocial needs of families of children with rare disease should be 
prioritized and they should be supported by genetic counselors throughout the process of testing, adaptation and coping [37].

Over recent years, more UKGCs have been elected to leadership positions and are influencing healthcare policy and education reform for example, NHS Clinical Reference Group Membership (which determines service specification for Clinical Genetics Services in England), committee and leadership roles within the European Society of Human Genetics, research roles (e.g., Society \& Ethics Research at the Wellcome Sanger Institute and academic positions associated with Institutions of Higher Education). An example of influencing policy has been the inclusion of UKGCs being able to directly order genomic tests through the new NHS Genomic Medicine Service test directory introduced in 2019 [38]. The AGNC contributed evidence to the Topol Review; an NHS review that considers the future of genomics in the NHS in the next 5-20 years. This advocates for more resources and support for genetic counsellors. The review reported that, "Counsellors and specialized physicians will have an extended role in training, provision of referral services, support for mainstreaming, and contributions to genomic leadership. They will have a leading role in the development of complex, ethical, patient-led management that encompasses an understanding of how genomic data can lead to a long-term duty of crae that extends beyond the index case to their wider families." (REF). This future vision includes many of the topics voiced by the genetic counsellors back in 2016. UKGCs have also been influential in the development of the bi-annual Wellcome Trust World Congress on Genetic Counseling and also hold roles within Health Education England and NHS England. The AGNC have endorsed a new free online training course, 'what is genetic counseling?' to help raise awareness of the profession and process of genetic counseling [39].

\section{Conclusion}

The research demonstrates that there was agreement among UKGCs that advances in genomic technology will change the way healthcare is delivered. Along with other health professional groups, genetic counseling will have to adapt accordingly. Participants recognized that it would be important to educate other professionals within the NHS, the public and assorted stakeholders about the contribution of genetic counselors and the professions' own USP. The findings from this empirical research show that as a profession, and as individual practitioners, this will require genetic counselors to KIDS certain aspects of their current remit. There is acknowledgment that this will be a joint responsibility: for the AGNC as a professional organization the GCRB as the professional regulator and for individual genetic counselors and also genetic counseling services.

Participants were enthusiastic about the things which were unique to the genetic counseling profession and the things they were good at and proud of. They felt that this deserved and required the development of a strategy with which to promote and market what is distinctive and valuable about their profession as a means of proactively positioning them to meet and capitalize on the challenges and opportunities in the era of genomic science. To this end, they acknowledged that this will require the profession to continuously review and redefine where necessary the purpose and remit of genetic counseling. There is evidence that since this research UKGCs have been active in engaging with policy development and embracing new roles. The evidence from this research will provide an important reference point for future research with genetic counselors in the UK NHS and a comparison with other countries and professions.

Financial \& competing interests disclosure

C Benjamin is employed by a Private Healthcare Company to provide Genetic Counseling. A Phillips is the Director of Alan Phillips Associates and as a Psychosocial Consultant provides Genetic counseling supervision and training to the profession. The authors have no other relevant affiliations or financial involvement with any organization or entity with a financial interest in or financial conflict with the subject matter or materials discussed in the manuscript apart from those disclosed.

No writing assistance was utilized in the production of this manuscript. 
- UK genetic counselors make up a very small proportion of the UK National Health Service healthcare workforce. However, their unique skillset will be pivotal in augmenting developments in the implementation of genomic technologies to improve patient care.

- The Association of Genetic Nurses and Counselors (AGNC) is the largest professional group representing genetic counselors in the UK and the Republic of Ireland. Their 2017 statement, 'The future role of genetic counselors in genomic healthcare' identifies the importance of continuing professional education and the need to respond to new ways of working.

- This paper describes empirical research undertaken at the 2016 AGNC conference, midway through the UK 100,000 Genomes Project and in anticipation of the publication of the Chief Medical Officer's 2016 Report 'Generation Genome'. It provides an insight into UK genetic counselors' opinions about the psychosocial component of their current remit and future opportunities and challenges.

- Data were collected from 60 participants using focus group methodology which was enhanced by two interactive group techniques; strengths, weaknesses, opportunities and threats, and keep, introduce, develop and stop. Discussion focused around the theme 'Psychosocial Issues in the New Genomics Era'.

- Genetic counselors recognized that the world of healthcare genetics/genomics is rapidly changing. They acknowledged that advances in technology and developments such as direct-to-consumer genetic testing, will require change to the genetic counseling remit and the role, rules, relationships and responsibilities that underpin it.

- Enthusiasm was generated regarding their particular specialist knowledge, emphasizing their unique selling point of skills in communication, counseling and psychosocial assessment. This included ideas on how they could respond to the changes and exercise as much influence as possible over the future direction of their profession.

- They acknowledged that other scientific and healthcare professionals were uncertain regarding the role and remit of genetic counselors and it was important to improve this and awareness of the profession.

- The focus group data were presented at the 2017 AGNC annual conference in the form of a poster summary in order to maintain momentum and to determine if any of the issues identified during the focus groups in 2016 had been achieved or were on-going.

- We report here an update of the recent literature and policy statements. This shows that significant achievements have been made since the workshop, for example, genetic counselors holding senior policy and leadership roles, as well as position statements and policy documents highlighting the genetic counselor role in genomic healthcare.

- To ensure that genomic technology leads to patient benefit, there needs to be an ongoing commitment of the whole healthcare multidisciplinary team, which includes the voice of genetic counselors.

\section{Ethical conduct of research}

The authors state that they have obtained appropriate institutional review board approval or have followed the principles outlined in the Declaration of Helsinki for all human or animal experimental investigations. In addition, for investigations involving human subjects, informed consent has been obtained from the participants involved.

\section{References}

Papers of special note have been highlighted as: • of interest

1. Association of Genetic Nurses and Counsellors (2019). http://www.agnc.org.uk/

2. Middleton A, Marks P, Bruce A et al. The role of genetic counsellors in genomic healthcare in the United Kingdom: a statement by the Association of Genetic Nurses and Counsellors. Eur. J. Hum. Genet. 25, 659-661 (2017).

- This is a pivitol statement by the UK professional association of genetic counselors and nurses. It discusses the current role of a UK genetic counselor and how this might adapt in the future due to increased genomic technologies. This provides a background to the research question posed in the submitted paper.

3. Turnbull C, Scott RH, Thomas E et al. The 100,000 Genomes Project: bringing whole genome sequencing to the NHS. BMJ 361, k1687 (2018).

4. Davies S. Chief Medical Officer annual report 2016: generation genome (2017). https://www.gov.uk/government/publications/chief-medical-officer-annual-report-2016-generation-genome

5. Dheensa S, Samuel G, Lucassen A, Farsides B. Towards a national genomics medicine service: the challenges facing clinical-research hybrid practices and the case of the 100,000 genomes project. J. Med. Ethics 44(6), 397-403 (2018).

- In this paper, which draws on 20 interviews with professional stakeholders involved in UK 100,000 Genomes Project, the authors investigate the ethical issues raised by this project's hybrid nature, that of provision of genomic clinical NHS testing and agrement that samples can be used for research purposes. This paper sets the political and ethical landscape facing UK genetic 
counselors' with new models of service delivery and other clinical specialties being able to access genomic testing witout the traditional gatekeeping role of the UK clinical genetics service.

6. Burton H, Hall A, Kroese M, Raza S. Genomics in mainstream clinical pathways (2017). http://www.phgfoundation.org/documents/PHGF-Genomics-in-mainstream-clinical-pathways.pdf

7. NHS England Clinical Reference Group Medical Genetics. Outline specification for the specialist commissioning of genetic and genomic services for England (2014). http://www.bsgm.org.uk/media/883258/bshg_july_51.pdf

8. The Kings Fund. Better value in the NHS: report summary (2015). https://www.kingsfund.org.uk/publications/better-value-nhs/summary

9. Rolewicz L, Palmer B. The NHS workforce in numbers. (2019). https://www.nuffieldtrust.org.uk/resource/the-nhs-workforce-in-numbers

10. NHS England (2019). https://www.england.nhs.uk/healthcare-science/what/

11. Association of Genetic Nurses and Counsellors (2019). https://www.agnc.org.uk/about-us/

12. Resta R, Biesecker BB, Bennett RL et al. A new definition of Genetic Counseling: National Society of Genetic Counselors' Task Force report. J. Genet. Couns. 15(2), 77-83 (2006).

13. Austin J, Semaka A, Hadjipavlou G. Conceptualizing genetic counseling as psychotherapy in the era of genomic medicine. J. Genet. Couns. 23(6), 903-909 (2014).

14. Biesecker B, Austin J, Caleshu C. Theories for psychotherapeutic genetic counseling: fuzzy trace theory and cognitive behavior theory. $J$. Genet Couns. 26(2), 322-330 (2017).

15. Redlinger-Grosse K. A different vantage point: commentary on "theories for psychotherapeutic genetic counseling: fuzzy trace theory and cognitive behavior theory”. J. Genet. Couns. 26(2), 331-333 (2017).

- Follows the recent US debate regarding the counseling element of genetic counseling and the issue of weather psychotheraputic counseling should be a practice discipline of genetic counseling.

16. British Association of Counsellors and Psychotherapists (2019). www.bacp.org.uk

17. UK Council for Psychotherapy (2019). www.ukcp.org.uk

18. Phillips A, Mannion G, Birch J. Crafting practice through supervision. Healthcare Couns. Psychother. J. 12(2), 30-36 (2012).

- Introduces the counseling supervision framework, consultative supervision and the 4Rs role, responsibility, responsibility and remit which is used by many UK genetic counselor teams.

19. Association of Genetic Nurses and Counsellors. Code of Ethics (2019). https://www.agnc.org.uk/info-education/documents-websites/

20. Winchester E, Limb S, Coleman C et al. The South West Thames Regional Genetic Service Hereditary Cancer Clinic: the expereince for the perspective of the patients and health professionals involved. World Congress Genet. Couns. 2017 (2017).

21. Wiggins J, Middleton A. Getting the Message Across: communicating with diverse populations in clinical genetics. Oxford University Press, Oxford, UK, (2013).

22. Purves D. Time-limited Practice. In: Handbook of Counselling Psychology. Woolfe R, Dryden W, Strawbridge S (Eds). Sage London, UK 532 (2003).

23. UK and Republic of Ireland Genetic Counsellor Registration Board (2019). http://www.gcrb.org.uk/

24. Calzone KA, Kirk M, Tonkin E, Badzek L, Benjamin C, Middleton A. The global landscape of nursing and genomics. J. Nurs. Scholarsh. 50(3), 249-256 (2018).

- Describes the global integration of genomic healthcare into all nursing practice and is the second in a series of papers produced by members of the G2NA (Global Genomics Nursing Alliance).

25. Ormond KE, Laurino MY, Barlow-Stewart K et al. Genetic counseling globally: where are we now? Am. J. Med. Genet. Part C: Semin Med. Genet. 178(1), 98-107 (2018).

26. Abacan M, Alsubaie L, Barlow-Stewart $\mathrm{K}$ et al. The global state of the genetic counseling profession. Eur. J. Hum. Genet. 27(2), 183-197 (2019).

- This paper is timely and provides data on the similarities and differences between countries concerning the development of the fairly recent healthcare profession of genetic counseling.

27. Patch C, Middleton A. Genetic counselling in the era of genomic medicine. British Medical Bulletin 126(1), 27-36 (2018).

- Provides a commentory on where they feel UK genetic counselors can provide important input into clinical services, research, policy and education agendas.

28. Krueger R, Casey M. Focus Groups. A Practical Guide for Applied Research. (4th Edition). Krueger R, Casey M (Eds). SAGE, London, UK, (2008).

29. Helms MM, Nixon J. Exploring SWOT analysis - where are we now? A review of academic research from the last decade. J. Strat. Manag. 3(3), 215-251 (2010). 
30. Wash M. 54 tools and techniques for business excellence. Publishers Management Books, Cirencester page 45 (2007). http://www.mb2000.com/acatalog-cgi-scripts/sh000001.pl?REFPAGE=http\%3a\%2f\%2fwww\%2emb2000\%2ecom\%2fac atalog\%2findex\%2ehtml\&WD=tools\%20techniques\%2054\&PN=Management\%2ehtml\%23a227\#a227

31. Phillips A. Alder hey assessed: Handling emotions at work. Healthcare Couns. Psychother. J. 7, 21-24 (2007).

32. Phillips A. Alder hey assessed: Light out of darkness. Healthcare Couns. Psychother. J. 6, 22-25 (2006).

- Provides the background to the deveopment of the Excellence through Learning (EtL) programme that was designed as the recovery and development programme for staff andpatient's at Alder Hey Children's Hospital following the Organ Retention scandal and the implementation of the recommendations in the Royal Liverpool Children's Inquiry; Published 30 January 2001: Ref: ISBN 9780102775013, HC 12 2000-01. This also details the use by this group of the keep, introduce, develop and stop model.

33. Perera R. The Pestel Analysis. Nerdynaut, Sri Lanka (2017). https://books.google.co.uk/books?id=ZWpLDwAAQBAJ

34. Krueger R, Casey M. Analysing focus group results, Chapter 6 Table 6.1. In: Focus Groups. A Practical Guide for Applied Research. Krueger R, Casey M (Eds). SAGE London, UK (2008).

35. National School of Healthcare Science. STP curriculum genomic counselling (2019). https://curriculum.nshcs.org.uk/programmes/stp/SLS4-2-18

36. Association of Genetic Nurses and Counsellors (2019). Job Profiles. http://www.agnc.org.uk/about-us/agnc-documents/job-profiles/

37. Joseph FM. A key role for the genetic counsellor in the genomics era. F1000Res. 7, 420-420 (2018).

38. NHS England. NHS genomic test directory (2019). https://www.england.nhs.uk/publication/national-genomic-test-directories/

39. Wellcome Genome Campus Advanced Courses and Scientific Conferences. What is genetic counselling? (2019). https://www.futurelearn.com/courses/what-is-genetic-counselling?utm_campaign=wellcome_genome_campus_what_is_genetic_counselli ng_june_2019\&utm_medium=organic_email\&utm_source=newsletter_broadcast\&dm_i=2SUU, W 18C,71NY4Y,3C2ST, 1

40. Health Education England. Topol E (2019).

https://url6.mailanyone.net/v1/?m=1iIUX1-0009ax-3c\&i=57e1b682\&c=cVhtnaniqLU8k_4wtkwAkmGjnTQlvrOSvrX6WxWPhoPJt 0Jijn-9TOtLnLtSwVLas2Iw-KOPPjrZUuLW58cOokG_AORPI6DdlHZQt9oTDIWBeqLtkm12moyX8b3eNM8473yrz2xMf 1xhjcd 06ibJaspD8sgHwV2_sFIv4fhi2TWYQ2C0pjf numh4Cs0pflWeMJLQ3PoLjckuRidV54dOI-Niq3C4pkz1-4SePCCacNo 